\title{
Higher circulating omentin is associated with increased risk of primary cardiovascular events in individuals with diabetes
}

\author{
Corinna Niersmann ${ }^{1,2} \cdot$ Maren Carstensen-Kirberg ${ }^{1,2} \cdot$ Haifa Maalmi $^{1,2} \cdot$ Bernd Holleczek $^{3} \cdot$ Michael Roden $^{1,2,4}$. \\ Hermann Brenner ${ }^{5,6}$. Christian Herder ${ }^{1,2,4}$ (D) $\cdot$ Ben Schöttker ${ }^{5,6}$
}

Received: 9 April 2019 / Accepted: 27 August 2019 / Published online: 9 November 2019

(C) Springer-Verlag GmbH Germany, part of Springer Nature 2019

\begin{abstract}
Aims/hypothesis Higher concentrations of the adipokine omentin are associated with lower levels of cardiometabolic risk factors in experimental and cross-sectional studies, but with higher risk of type 2 diabetes and cardiovascular diseases in population-based cohort studies. However, it is unknown whether high omentin concentrations are associated with increased risk of cardiovascular events in people with established diabetes. Therefore, the present study investigated the association between serum omentin concentrations and the risk of cardiovascular events in individuals with diabetes.

Methods This prospective study was based on participants of the German ESTHER cohort with diabetes and without previous cardiovascular event. The ESTHER cohort consists of individuals aged 50-75 years at baseline who were recruited by their general practitioners. After exclusion of individuals with serum C-reactive protein $\geq 10 \mathrm{mg} / 1$ ( $\geq 95.24 \mathrm{nmol} / \mathrm{l})$, the final analysis population consisted of 933 individuals. At baseline, serum omentin concentrations were measured by ELISA. Cox regression models were fitted to estimate HRs and their corresponding 95\% CIs for associations of omentin tertiles with a composite endpoint of cardiovascular events and separately with incident myocardial infarction, stroke and cardiovascular death.

Results During 14 years of follow-up, 228 individuals experienced a primary cardiovascular event (myocardial infarction, stroke or cardiovascular death). After comprehensive adjustment for age, sex, BMI, metabolic and lifestyle factors and medication use, HRs $(95 \% \mathrm{CIs})$ for the $2 \mathrm{nd}$ and 3 rd tertile of omentin compared with the 1 st tertile were: $1.24(95 \% \mathrm{CI} 0.86,1.79)$ and $1.63(1.15,2.32)\left(p_{\text {trend }}\right.$ $=0.005)$ for the composite cardiovascular endpoint; $1.39(0.78,2.47)$ and $1.71(0.98,2.99)\left(p_{\text {trend }}=0.065\right)$ for incident myocardial infarction; $1.40(0.78,2.53)$ and $2.05(1.17,3.58)\left(p_{\text {trend }}=0.010\right)$ for incident stroke; and $1.43(0.85,2.40)$ and $1.72(1.04,2.83)\left(p_{\text {trend }}=\right.$ 0.040) for cardiovascular death. Effect estimates and $p$ values were almost unaltered after additional adjustment for adiponectin.

Conclusions/interpretation Higher omentin concentrations are associated with an increased risk for cardiovascular events in individuals with diabetes after adjustment for multiple cardiovascular risk factors. Given data from preclinical studies, it appears possible that this association reflects a compensatory, but insufficient upregulation of omentin concentrations as a response to stimuli that increase cardiovascular risk.
\end{abstract}

Keywords Adipokine $\cdot$ Cardiovascular $\cdot$ Cohort study $\cdot$ Diabetes $\cdot$ Omentin

Corinna Niersmann and Maren Carstensen-Kirberg are joint first authors. Christian Herder and Ben Schöttker are joint senior authors.

Electronic supplementary material The online version of this article (https://doi.org/10.1007/s00125-019-05017-2) contains peer-reviewed but unedited supplementary material, which is available to authorised users.

Christian Herder

christian.herder@ddz.de

1 Institute for Clinical Diabetology, German Diabetes Center, Leibniz Center for Diabetes Research at Heinrich Heine University Düsseldorf, Auf'm Hennekamp 65, 40225 Düsseldorf, Germany

2 German Center for Diabetes Research (DZD), München-Neuherberg, Germany
3 Saarland Cancer Registry, Saarbrücken, Germany

4 Division of Endocrinology and Diabetology, Medical Faculty, Heinrich Heine University, Düsseldorf, Germany

5 Division of Clinical Epidemiology and Aging Research, German Cancer Research Center, Heidelberg, Germany

6 Network Aging Research, University of Heidelberg, Heidelberg, Germany 


\section{Research in context}

\section{What is already known about this subject?}

- Data from experimental studies point towards cardioprotective effects of omentin

- Cross-sectional studies showed inverse associations between omentin concentration and cardiometabolic risk

- By contrast, prospective studies showed positive associations between omentin and both incident type 2 diabetes and incident cardiovascular events in population-based cohorts

\section{What is the key question?}

- Are systemic omentin concentrations associated with the risk of cardiovascular outcomes in individuals with diabetes?

What are the new findings?

- Data from the ESTHER study show for the first time that higher serum concentrations of omentin are associated with a higher risk for primary cardiovascular events (composite endpoint based on myocardial infarction, stroke or cardiovascular death) in individuals with diabetes

- The association remains robust after comprehensive adjustment for age, sex, BMI, metabolic and lifestyle factors and medication use

- Among the separate cardiovascular outcomes, higher omentin concentrations are significantly associated with a higher risk of stroke and cardiovascular death

How might this impact on clinical practice in the foreseeable future?

- The potential role of omentin in the development of cardiovascular disease, and stroke in particular, merits further studies to clarify the relevance of this adipokine in the pathogenesis of these outcomes

\section{Abbreviations \\ CRP C-reactive protein \\ GP General practitioner \\ IQR Interquartile range \\ NSAID Nonsteroidal anti-inflammatory drug}

\section{Introduction}

Omentin (also known as omentin-1 or intelectin-1) was initially discovered as adipokine from the stromal-vascular fraction of visceral adipose tissue $[1,2]$, but is also expressed by other tissues such as lung, heart and placenta, as shown recently [3]. Whereas several studies reported inverse associations of omentin concentrations with BMI and/or waist circumference [4, 5], such associations could not be observed at all [6-9] or after multivariable adjustment [10, 11] in other study populations. Early cross-sectional studies reported that higher systemic omentin concentrations were associated with higher adiponectin concentrations, higher insulin sensitivity, lower prevalence of type 2 diabetes and lower levels of multiple cardiovascular risk factors [4, 10-14]. Studies using mouse models pointed towards atheroprotective properties of omentin [15-17].
In contrast, longitudinal studies found that higher systemic omentin concentrations were associated with an increased risk of type 2 diabetes and with higher cardiovascular event rates in both population-based cohorts and in individuals with preexisting cardiovascular diseases [5, 9, 18, 19].

Given the increased cardiovascular risk in individuals with type 2 diabetes, it is of interest to identify biomarkers that predict cardiovascular events in this subgroup of the population. Several studies have implicated inflammation-related biomarkers such as IL-6 and IL-15 as potential risk factors $[20,21]$, but it is not known yet whether higher omentin concentrations are associated with a higher risk of cardiovascular events in individuals with type 2 diabetes.

Therefore, this study aimed to assess the association between serum omentin concentrations and incident cardiovascular events (myocardial infarction, stroke and cardiovascular death; composite endpoint defined as the first of these cardiovascular events) in individuals with diabetes. Additionally, we investigated whether such associations were independent of adiponectin.

\section{Methods}

Study population This investigation is based on the ESTHER study (German study name: Epidemiologische Studie zu 
Chancen der Verhütung, Früherkennung und optimierten THerapie chronischer ERkrankungen in der älteren Bevölkerung), an ongoing cohort study including 9949 people in the age range of 50-74 years at baseline [22]. The investigations were carried out in accordance with the Declaration of Helsinki, including written informed consent from all participants. The study was approved by the ethics committees of the Medical Faculty, University of Heidelberg (Heidelberg, Germany) and the Medical Association of Saarland (Saarbrücken, Germany). Study participants were recruited by their general practitioners (GPs) during a routine health check-up between 2000 and 2002 in Saarland, a federal state of Germany [23, 24]. The distributions of baseline characteristics such as demographic variables, socioeconomic variables and prevalence of common chronic diseases were comparable to those in the population-based German National Health Survey when comparing the same age range [23].

This analysis was limited to 1375 individuals with physician-diagnosed diabetes at baseline (based on a documented diagnosis of diabetes by the treating physician or based on the use of prescribed glucose-lowering drugs). From these study participants, we excluded individuals: (1) who were diagnosed with diabetes before the age of 40 years ( $n=53$; because of potential type 1 diabetes); (2) with missing serum sample or insufficient serum volume for omentin measurements $(n=45)$; (3) with self-reported history of myocardial infarction or stroke at baseline $(n=211)$; or (4) with serum concentrations of C-reactive protein (CRP) $\geq 10 \mathrm{mg} / \mathrm{l}$ equivalent to $\geq 95.24 \mathrm{nmol} / 1$ ( $n=96$; because of potential acute infections or tissue damage). In addition, we excluded participants lost to follow-up $(n=37)$, leaving 933 individuals for the current analysis. Given the low population prevalence of type 1 diabetes, the vast majority of the study participants can be assumed to have type 2 diabetes.

The collection of baseline data on anthropometric, sociodemographic, metabolic and lifestyle factors as well as information on the presence of chronic diseases (including cardiovascular events) and on medication use were described in detail elsewhere [20, 25]. Study participants were recorded as having hypertension if this was documented on the health check-up form by the treating physician or if antihypertensive drugs were prescribed. The eGFR was calculated based on the Chronic Kidney Disease Epidemiology Collaboration (CKD-EPI) equation: eGFR $=127.7$ [cystatin $\mathrm{C}(\mathrm{mg} / \mathrm{l})]^{-1.17} \times$ age $^{-0.13} \times$ [0.91 if female] [1.06 if black]) [26]. Serum concentrations of CRP and adiponectin were measured by immunoturbidimetry with the wrCRP antibody (Bayer, Leverkusen, Germany) on the ADVIA 2400 (Siemens Healthcare Diagnostics, Eschborn, Germany) and by ELISA, respectively [20].

Definition of cardiovascular outcomes The definition of cardiovascular outcomes in the ESTHER study has been described previously $[20,25]$. Briefly, study participants reported incident myocardial infarctions and strokes in standardised questionnaires during the follow-up examinations at 2, 5, 8, 11 and 14 years. At the 2- and 5-year followup, validation of self-reported myocardial infarctions and strokes was done by sending short questionnaires to the GPs asking them to confirm that such an event has taken place in the last 2 or 3 years. At the 8-, 11- and 14-year follow-up all GPs of the study participants were sent questionnaires, which included, among other things, questions about myocardial infarctions and strokes in the past 3 years. The cardiovascular events were only taken into account in the analysis if they were confirmed by the GP. If the GP did not send back a questionnaire, the self-reported information was used. However, this was rarely necessary because we were able to validate $94 \%$ of the events used for the composite outcome of cardiovascular events. We asked the GPs and the study participants to give exact dates of the cardiovascular events and these were used in the analyses. Only in the rare event that no date was given, the questionnaire return date was used as a proxy for the event date. Information on vital status at the end of the year 2015 was obtained from a central register of residents' registration offices of Saarland. The leading cause of death of deceased study participants was retrieved from death certificates obtained from public health offices in Saarland. Deaths coded with International Statistical Classification of Diseases and Related Health Problems (ICD)-10 codes I21I23 and I60-I69 were recorded deaths due to myocardial infarctions and strokes, respectively. All deaths coded with ICD10 codes I00-I99 were considered as cardiovascular-specific deaths. A composite endpoint of cardiovascular events was defined based on the first occurrence of an incident myocardial infarction, an incident stroke or cardiovascular death in accordance with previous analyses on cardiovascular outcomes in this cohort $[20,25]$. The rationale for the definition of such a composite endpoint is based on the assumption that associations between omentin and the three constituent outcomes are similar due to overlaps in their pathophysiology.

Quantification of omentin serum concentrations Omentin concentrations were assessed in serum samples that had been collected at baseline between 2000 and 2002 and stored at $-80^{\circ} \mathrm{C}$ until analysis in June 2018. Serum omentin concentrations were quantified using the Omentin-1 Human ELISA (BioVendor, Brno, Czech Republic) as previously described $[11,27]$. Intra- and inter-assay CVs were $3.4 \%$ and $7.1 \%$, respectively.

Statistical analysis Baseline characteristics are given as median (interquartile range [IQR]) for continuous variables and as absolute numbers and percentages for categorical variables. Differences between omentin tertiles were analysed with the Wilcoxon test for continuous variables, whereas the $\chi^{2}$ test 
was used for categorical variables. The association between omentin and adiponectin concentrations was assessed using multivariable linear regression analysis.

Cumulative incidences for all outcomes were visualised using Kaplan-Meier plots stratified by tertiles of omentin concentrations. Differences between cumulative incidences for omentin tertiles were tested for statistical significance using logrank test.

Cox proportional hazards models were fitted to estimate HRs and $95 \%$ CIs for the association between tertiles of omentin and cardiovascular endpoints. Trend tests $\left(p_{\text {trend }}\right)$ were estimated using the median of each omentin tertile. In addition, analyses were carried out per doubling of omentin using $\log _{2}$-transformed omentin as an independent variable to allow the comparison with a previous report [5].

Covariates of the models were selected a priori from factors potentially associated with cardiovascular risk, omentin and other adipokines based on previous studies [19, 20, 28-30]. Four models with increasing adjustment were built. In model 1 , analyses were adjusted for age and sex. In model 2 , analyses were additionally adjusted for smoking and physical activity as the most relevant lifestyle factors. In model 3, which should be considered our fully adjusted model, analyses were further adjusted for BMI $\left(\mathrm{kg} / \mathrm{m}^{2}\right), \mathrm{HbA}_{1 \mathrm{c}}(\%)$, total cholesterol (mg/ $\mathrm{dl})$, eGFR ( $\mathrm{ml} \mathrm{min}^{-1}[1.73 \mathrm{~m}]^{2}$ ), hypertension (yes/no), use of glucose-lowering medication (yes/no), use of statins (yes/no), use of antihypertensive drugs (yes/no) and use of nonsteroidal anti-inflammatory drugs (NSAIDs, yes/no). In model 4, we additionally adjusted for adiponectin $(\mu \mathrm{g} / \mathrm{ml})$ to evaluate the extent to which the fully adjusted estimates are independent of adiponectin. Based on results from a previous study [11] we hypothesised that adiponectin might be a mediator between omentin and cardiovascular disease risk rather than a potential confounder.

To explore potential effect modification, we performed subgroup analyses for model 3 stratified by sex and also by defined cut-off values for age ( $\geq 65$ vs $<65$ years), BMI ( $\geq 30$ vs $<30 \mathrm{~kg} / \mathrm{m}^{2}$ ), CRP ( $\geq$ median vs $<$ median) and adiponectin ( $\geq$ sex-specific median vs $<$ sex-specific median) to ensure meaningful sizes of the subgroups. $P$ values for interaction of omentin tertiles and subgroups were obtained after including a multiplicative term into the Cox proportional hazards regression model.

To explore potential differences in effect sizes depending on the time of event during the follow-up, we repeated our main analysis (model 3) and calculated HRs for study participants who experienced their outcomes within the first 4 years of follow-up (length of follow-up chosen so that subgroups had at least 20 events) and those who experienced their outcomes later during the follow-up.

All statistical analyses were performed with SAS 9.4 (SAS Institute, Cary, NC, USA). A $p$ value $<0.05$ was considered to indicate statistical significance.

\section{Results}

Baseline characteristics of the study population Table 1 shows the baseline characteristics of the study population $(n=933)$ stratified by tertiles of serum omentin concentrations. Individuals with higher omentin concentrations were older, more likely to be female, less likely to be current smokers, had lower concentrations of total cholesterol, lower eGFR, differed in their use of glucose-lowering medication (higher proportion of insulin users in top tertile) and statins (less frequent use in top tertile) and differed in their adiponectin concentrations (without consistent trend over the tertiles). No difference was observed for physical activity, BMI, $\mathrm{HbA}_{1 \mathrm{c}}$, the prevalence of hypertension, the use of antihypertensive drugs, the use of NSAIDs and for CRP concentrations.

We performed a multivariable linear regression analysis to assess the association between omentin and adiponectin concentrations in more detail. After adjustment for age and sex, this association was inverse $(\beta[95 \% \mathrm{CI}]-3.8[-6.8,-0.8]$, $p=0.01$ ). However the association was abolished by full adjustment for age, sex, smoking, physical activity, $\mathrm{BMI}, \mathrm{HbA}_{1 \mathrm{c}}$, total cholesterol, eGFR, hypertension, use of glucose-lowering medication, use of statins, use of antihypertensive drugs and use of NSAIDs ( $\beta[95 \% \mathrm{CI}]-0.9[-4.0,2.2], p=0.55$ ).

Incidence of cardiovascular events During 14 years of followup, 85 study participants had an incident primary myocardial infarction $(9.1 \%$; incidence rate $=10.1$ per 1,000 personyears), 96 had a stroke $(10.3 \%$, incidence rate $=11.5$ per 1,000 person-years), and 119 had a fatal cardiovascular event $(12.7 \%$, incidence rate $=10.1$ per 1000 person-years $)$, leading to 228 individuals who reached the composite cardiovascular endpoint that only recorded first events in the case of multiple events $(24.4 \%$, incidence rate $=27.5$ per 1000 person-years $)$. Cumulative incidences for all outcomes stratified by tertiles of omentin concentration were visualised using Kaplan-Meier plots (electronic supplementary material [ESM] Fig. 1). Compared with individuals who did not experience a cardiovascular event during the 14-year follow-up, study participants with incident cardiovascular event had higher omentin concentrations (median [IQR] 448.7 [348.0-590.7] vs 396.2 [328.0-505.9] ng/ml, $p<0.001)$.

Multivariable association between serum omentin and cardiovascular outcomes Omentin concentrations showed a concentration-dependent association with the incidence of the composite outcome of any cardiovascular event as well as with the incidence of stroke and cardiovascular death (Table 2) after adjustment for age and sex (model $1, p_{\text {trend }} \leq$ 0.005). Effect estimates were hardly changed after additional adjustment for lifestyle factors (model $2, p_{\text {trend }} \leq 0.003$ ) and slightly attenuated in the fully adjusted model 3 ( $p_{\text {trend }} \leq$ 0.040). Fully adjusted HRs ( $95 \%$ CIs) for the comparison of 
Table 1 Baseline characteristics of the study population stratified by tertiles of serum concentrations of omentin

\begin{tabular}{|c|c|c|c|c|c|}
\hline Baseline characteristic & $\begin{array}{l}\text { Total sample } \\
n=933 \\
n(\%) \text { or median (IQR) }\end{array}$ & $\begin{array}{l}\text { Tertile } 1(\leq 357.5 \mathrm{ng} / \mathrm{ml}) \\
n=311 \\
n(\%) \text { or median }(\mathrm{IQR})\end{array}$ & $\begin{array}{l}\text { Tertile } 2(357.8-474.1 \mathrm{ng} / \mathrm{ml}) \\
n=311 \\
n(\%) \text { or median }(\mathrm{IQR})\end{array}$ & $\begin{array}{l}\text { Tertile } 3(>474.1 \mathrm{ng} / \mathrm{ml}) \\
n=311 \\
n(\%) \text { or median }(\mathrm{IQR})\end{array}$ & $p$ \\
\hline \multicolumn{6}{|l|}{ Demographic } \\
\hline Age (years) & $64(60-69)$ & $63(58-67)$ & $64(60-69)$ & $65(61-70)$ & $<0.001$ \\
\hline Female sex & $456(48.9)$ & $130(41.8)$ & $154(49.5)$ & $172(55.3)$ & 0.003 \\
\hline \multicolumn{6}{|l|}{ Lifestyle } \\
\hline Current smoking ${ }^{\mathrm{a}}$ & $144(16.0)$ & $61(20.2)$ & $40(13.4)$ & $43(14.4)$ & 0.049 \\
\hline Low physical activity ${ }^{\mathrm{a}}$ & $672(72.6)$ & $232(75.0)$ & $211(68.9)$ & $229(73.9)$ & 0.196 \\
\hline \multicolumn{6}{|l|}{ Clinical } \\
\hline BMI $\left(\mathrm{kg} / \mathrm{m}^{2}\right)^{\mathrm{a}}$ & $29.0(26.6-31.9)$ & $29.1(26.5-32.4)$ & $29.1(26.8-31.8)$ & $28.7(26.4-31.6)$ & 0.265 \\
\hline $\mathrm{HbA}_{1 \mathrm{c}}(\mathrm{mmol} / \mathrm{mol})^{\mathrm{a}}$ & $50(42-60)$ & $50(43-57)$ & $49(42-57)$ & $51(42-62)$ & 0.173 \\
\hline $\mathrm{HbA}_{1 \mathrm{c}}(\%)^{\mathrm{a}}$ & $6.7(6.0-7.6)$ & $6.7(6.1-7.4)$ & $6.6(6.0-7.4)$ & $6.8(6.0-7.8)$ & 0.173 \\
\hline Total cholesterol (mmol/l) & $5.56(4.64-6.38)$ & $5.69(4.83-6.39)$ & $5.71(4.98-6.50)$ & $4.98(3.95-6.08)$ & $<0.001$ \\
\hline eGFR $\left(\mathrm{ml} \mathrm{min}{ }^{-1}[1.73 \mathrm{~m}]^{2}\right)^{\mathrm{a}}$ & $78.2(65.9-90.1)$ & $81.6(70.7-91.8)$ & $77.7(65.7-90.7)$ & $74.6(61.5-86.9)$ & $<0.001$ \\
\hline Hypertension & $689(73.8)$ & $220(70.7)$ & $230(73.9)$ & $239(76.8)$ & 0.203 \\
\hline \multicolumn{6}{|l|}{ Medication } \\
\hline $\begin{array}{l}\text { Glucose-lowering } \\
\text { medication }\end{array}$ & & & & & 0.045 \\
\hline No medication & $454(48.7)$ & $150(48.2)$ & $168(54.0)$ & $136(43.7)$ & \\
\hline Oral medication & $340(36.4)$ & $119(38.2)$ & $105(33.8)$ & $116(37.3)$ & \\
\hline Insulin & $139(14.9)$ & $42(13.5)$ & $38(12.2)$ & $59(18.9)$ & \\
\hline Statins & $81(8.7)$ & $23(7.4)$ & $40(12.9)$ & $18(5.8)$ & 0.005 \\
\hline Antihypertensive drugs & $578(61.9)$ & $186(59.8)$ & $194(62.4)$ & $198(63.7)$ & 0.601 \\
\hline NSAIDs & $213(22.8)$ & $66(21.2)$ & $72(23.1)$ & $75(24.1)$ & 0.682 \\
\hline \multicolumn{6}{|l|}{ Biomarker } \\
\hline Omentin (ng/ml) & $407.7(331.5-527.1)$ & $305.5(267.2-331.5)$ & $407.7(379.1-441.5)$ & $598.2(527.1-772.6)$ & $<0.001$ \\
\hline Adiponectin $(\mu \mathrm{g} / \mathrm{ml})$ & $7.6(4.6-10.7)$ & $7.2(4.9-9.9)$ & $8.3(5.2-11.3)$ & $6.7(3.9-11.1)$ & 0.015 \\
\hline CRP (nmol/l) & $22(11-43)$ & $22(12-44)$ & $21(11-42)$ & $21(10-43)$ & 0.604 \\
\hline
\end{tabular}

Data are given as median (IQR) or absolute number $n$ (percentage)

${ }^{\mathrm{a}}$ The following variables had missing values: smoking $(n=34)$; physical activity $(n=8)$; $\mathrm{BMI}(n=3) ; \operatorname{HbA}_{1 \mathrm{c}}(n=2)$; eGFR $(n=4)$

$p$ values for differences between omentin tertiles were obtained using the Kruskal-Wallis rank test for continuous variables and using the $\chi^{2}$ test for categorical variables

omentin concentrations in tertile 3 vs tertile 1 were $1.63(1.15$, 2.32) for the incidence of any cardiovascular event, 2.05 $(1.17,3.58)$ for the incidence of stroke and $1.72(1.04,2.83)$ for cardiovascular death. For incident myocardial infarction, there was a trend towards an association with higher omentin concentrations, which was only significant for model 2 ( $p_{\text {trend }}$ $=0.044$ ). Inclusion of study participants with elevated CRP levels $\geq 10 \mathrm{mg} / \mathrm{l}(95.24 \mathrm{nmol} / \mathrm{l})$ led to similar results in model 3 showing associations of omentin concentrations with the composite outcome as well as with the incidence of stroke and cardiovascular death (all $p_{\text {trend }} \leq 0.03$ ), but not with incident myocardial infarction $\left(p_{\text {trend }}=0.23\right)$.

When adiponectin was considered as an additional covariable (model 4 ), HRs and $p_{\text {trend }}$ for the associations between omentin and all four cardiovascular outcomes remained almost unchanged (Table 2).

When associations were estimated per doubling of omentin (model 3), HRs (95\% CIs) were 1.39 (1.08, 1.81) for incident cardiovascular events $(p=0.011), 1.44(0.95,2.18)$ for incident myocardial infarction $(p=0.084), 1.66(1.10,2.48)$ for incident stroke $(p=0.014)$ and $1.40(0.99,1.98)$ for cardiovascular death $(p=0.057)$.

For the outcomes cardiovascular events, stroke and myocardial infarction, subgroup analyses did not show any significant effect modification across strata of sex, age, BMI, CRP and adiponectin (ESM Table 1). However, for cardiovascular death, while no significant effect modification was detected by sex, age and BMI, higher HRs were observed in subgroups 
Table 2 Association between serum omentin (tertiles) and cardiovascular outcomes

\begin{tabular}{|c|c|c|c|c|}
\hline & \multicolumn{3}{|c|}{ Omentin (HR and $95 \% \mathrm{CI}$ ) } & \multirow[b]{2}{*}{$p_{\text {trend }}$} \\
\hline & Tertile 1 & Tertile 2 & Tertile 3 & \\
\hline \multicolumn{5}{|c|}{ Cardiovascular event } \\
\hline$n$ at risk & 298 & 294 & 297 & \\
\hline$n$ of events & 56 & 67 & 94 & \\
\hline Model 1 & Reference & $1.27(0.89,1.80)$ & $1.83(1.31,2.54)$ & $<0.001$ \\
\hline Model 2 & Reference & $1.33(0.93,1.90)$ & $1.87(1.34,2.62)$ & $<0.001$ \\
\hline Model 3 & Reference & $1.24(0.86,1.79)$ & $1.63(1.15,2.32)$ & 0.005 \\
\hline Model 4 & Reference & $1.22(0.84,1.76)$ & $1.65(1.16,2.34)$ & 0.004 \\
\hline \multicolumn{5}{|c|}{ Incident myocardial infarction } \\
\hline$n$ at risk & 298 & 294 & 297 & \\
\hline$n$ of events & 22 & 27 & 34 & \\
\hline Model 1 & Reference & $1.28(0.73,2.23)$ & $1.64(0.96,2.80)$ & 0.069 \\
\hline Model 2 & Reference & $1.39(0.79,2.45)$ & $1.76(1.02,3.03)$ & 0.044 \\
\hline Model 3 & Reference & $1.39(0.78,2.47)$ & $1.71(0.98,2.99)$ & 0.065 \\
\hline Model 4 & Reference & $1.36(0.76,2.42)$ & $1.74(0.99,3.03)$ & 0.055 \\
\hline \multicolumn{5}{|l|}{ Incident stroke } \\
\hline$n$ at risk & 298 & 294 & 297 & \\
\hline$n$ of events & 20 & 27 & 42 & \\
\hline Model 1 & Reference & $1.44(0.82,2.52)$ & $2.22(1.31,3.74)$ & 0.002 \\
\hline Model 2 & Reference & $1.46(0.81,2.60)$ & $2.26(1.32,3.87)$ & 0.002 \\
\hline Model 3 & Reference & $1.40(0.78,2.53)$ & $2.05(1.17,3.58)$ & 0.010 \\
\hline Model 4 & Reference & $1.40(0.78,2.53)$ & $2.04(1.17,3.57)$ & 0.010 \\
\hline \multicolumn{5}{|c|}{ Cardiovascular death } \\
\hline$n$ at risk & 298 & 294 & 297 & \\
\hline$n$ of events & 26 & 36 & 52 & \\
\hline Model 1 & Reference & $1.46(0.88,2.40)$ & $1.98(1.23,3.19)$ & 0.005 \\
\hline Model 2 & Reference & $1.54(0.93,2.57)$ & $2.05(1.27,3.31)$ & 0.003 \\
\hline Model 3 & Reference & $1.43(0.85,2.40)$ & $1.72(1.04,2.83)$ & 0.040 \\
\hline Model 4 & Reference & $1.37(0.82,2.30)$ & $1.71(1.04,2.82)$ & 0.036 \\
\hline
\end{tabular}

Model 1: adjusted for age and sex

Model 2: additionally adjusted for smoking and physical activity

Model 3: additionally adjusted for BMI, $\mathrm{HbA}_{1 \mathrm{c}}$, total cholesterol, eGFR, hypertension, use of glucose-lowering medication, use of statins, use of antihypertensive drugs, use of NSAIDs

Model 4: additionally adjusted for adiponectin with lower CRP concentrations $\left(p_{\text {interaction }}=0.03\right)$ and higher adiponectin concentrations $\left(p_{\text {interaction }}=0.001\right)$.

As shown in ESM Table 2, HRs were larger for all four outcomes for study participants who experienced their events within the first 4 years during the followup compared with those who had their events later during the follow-up.

\section{Discussion}

This study shows that higher systemic omentin concentrations were associated with a higher incidence of primary cardiovascular events, a higher incidence of primary stroke and a higher incidence of cardiovascular death in individuals with diabetes. These associations were independent of adiponectin concentrations.

Association between omentin and cardiovascular outcomes Our data are novel because previous studies on omentin and cardiovascular risk focused on individuals from the general population [5] or on patients undergoing coronary angiography due to established or suspected coronary artery disease [9], whereas data for individuals with diabetes were not available. This study extends the current literature by demonstrating that higher omentin concentrations were associated with a higher risk of primary cardiovascular events in individuals with diabetes. 
In accordance with our previous analysis within the ESTHER cohort [20] we used a composite cardiovascular endpoint as main outcome. In addition, the increased number of individuals with incident cardiovascular events due to the longer follow-up time of 14 years also allowed for a meaningful analysis of myocardial infarction, stroke and cardiovascular death as separate entities. Whereas a doubling of omentin was associated with incident stroke (HR [95\% CI] $2.22[1.52,3.22])$ but not with incident myocardial infarction (HR 1.17 [0.79, 1.72]) in the population-based European Prospective Investigation into Cancer and Nutrition (EPIC)-Potsdam cohort [5], the effect estimates for both outcomes in our study were more similar and intermediate between those associations. Of note, effect estimates for incident stroke were lower in the EPIC-Potsdam cohort for individuals with the metabolic syndrome or any of its components compared with metabolically healthy individuals, which is in line with the lower HR in the ESTHER cohort comprising only people with diabetes compared with the HR from the EPIC-Potsdam sample. In another study on individuals with pre-existing cardiovascular disease, omentin was positively associated with different composite cardiovascular endpoints, but incident stroke or myocardial infarction were not analysed separately due to the insufficient number of individuals with incident events [9]. Taken together, higher omentin concentrations were associated with a higher risk of cardiovascular events in three cohorts with different baseline characteristics and outcome definitions.

\section{Potential explanations for the positive association between} omentin and cardiovascular risk The majority of experimental studies points towards favourable effects of omentin on cardiometabolic risk. Preclinical studies using mouse models of atherosclerosis or ischaemia/reperfusion injury demonstrated that systemic administration or overexpression of omentin delayed the development of aortic atherosclerotic lesions and reduced myocardial infarct size [15-17]. Additionally, lentiviral overexpression of omentin reduced the brain infarction volume in a mouse model of ischaemic stroke [31]. In vitro studies investigating the impact of omentin on endothelial cells, smooth muscle cells and macrophages suggested atheroprotective properties reflected by reduced monocyte adhesion, improved cell differentiation and survival rate, enhanced vasodilation and attenuation of inflammatory processes [32,33], although omentin treatment appeared to have proinflammatory effects on adipocytes [34].

In line with the preclinical studies, cross-sectional studies reported that circulating omentin concentrations were lower in people with coronary artery disease or ischaemic stroke compared with individuals without cardiovascular disease $[10$, 35-40]. Lower omentin concentrations were also related to less favourable short-term functional outcome in individuals with ischaemic stroke [39]. These data are in agreement with observations from several studies showing that omentin concentrations were inversely correlated with multiple cardiovascular risk factors including age, BMI, waist circumference, glucose levels, insulin resistance and lipid levels $[4,5,11,41]$. However, other studies also found omentin to be largely independent of cardiovascular risk factors [4, 7-9]. Data from the ESTHER study are also not able to confirm inverse associations of omentin with $\mathrm{BMI}$ or $\mathrm{HbA}_{1 \mathrm{c}}$. The potential reasons for this discrepancy are currently unclear.

However, in contrast to preclinical and cross-sectional studies, prospective studies show that higher omentin concentrations are associated with increased cardiovascular risk. Higher omentin concentrations in individuals at risk for cardiovascular events may reflect a counterregulatory mechanism. Omentin could be upregulated in response to metabolic and inflammatory stimuli that contribute to atherogenesis, but this appears insufficient to protect against the onset of cardiovascular events. If this were true, associations could be explained by reverse causation because higher biomarker levels would represent early symptoms of cardiovascular disease. Although our subgroup analyses stratified by sex, age, BMI, CRP and adiponectin argue against differences in associations according to these variables, our observations of stronger associations between omentin and cardiovascular events occurring early during the follow-up support this interpretation.

In this respect, omentin may belong to a growing group of biomarkers including adiponectin, growth differentiation factor 15 (GDF-15), IL-1 receptor antagonist (IL-1 ra) or IL22 that appear to have cardioprotective effects in experimental studies, but nevertheless show a positive association with cardiovascular risk in multiple cohorts [29, 42-47].

Strengths and limitations Strengths of this study include the selection of individuals with diabetes from a cohort that is representative for this patient group in Germany, the prospective design, the long follow-up, the analysis of different cardiovascular outcomes and the comprehensive adjustment for cardiometabolic risk factors. Limitations comprise the fact that omentin was measured at only one time point, whereas serial biomarker measurement may allow more precise risk estimation [48]. Our study population most likely consisted mainly of individuals with type 2 diabetes. In the absence of autoantibody measurements, we assumed that individuals with a diagnosis of diabetes before the age of 40 years may have type 1 diabetes, which would have led to selective exclusion of some participants with young-onset type 2 diabetes and rare inclusion of individuals with type 1 diabetes. Data on waist circumference were not collected in the ESTHER study. We therefore could not adjust for this measure of abdominal obesity. Moreover, our study included older individuals aged 50-74 years of mainly German descent. Thus, our results may not be generalisable to younger 
populations and to populations with non-European descent. Finally, serum samples were stored for 16-18 years before analysis, which could have affected absolute omentin concentrations in our measurements. However, there is no reason to assume that this could have introduced a differential bias in our analysis since serum samples for both participants with and without events had the same storage conditions.

Conclusion Higher omentin concentrations were associated with a higher risk of primary cardiovascular events, stroke and cardiovascular death in individuals with diabetes. Several lines of evidence suggest that omentin may not be a genuine cardiovascular risk factor and that the upregulation of systemic omentin concentrations before the onset of cardiovascular events may reflect an anti-inflammatory counterregulation, which, however, is not pronounced enough to confer cardiovascular protection.

Acknowledgements Data from this study were presented as an abstract at the Diabetes Congress of the German Diabetes Association (DDG) in Berlin, Germany, 29 May-1 June 2019 and at the 55th Annual Meeting of the European Association for the Study of Diabetes (EASD) in Barcelona, Spain, 16-20 September 2019.

Data availability The data are subject to national data protection laws and restrictions were imposed by the ethics committees of the Medical Faculty, University of Heidelberg and the Medical Association of Saarland to ensure data privacy of the study participants. Therefore, data cannot be made freely available in a public repository. However, data can be requested through an individual project agreement with ESTHER. Please contact H. Brenner (principal investigator of the ESTHER study) in case of further questions.

Funding The ESTHER study was funded by the Saarland state Ministry for Social Affairs, Health, Women and Family Affairs (Saarbrücken, Germany), the Ministry of Science, Research and Arts of the state of Baden-Württemberg (Stuttgart, Germany), the German Federal Ministry of Education and Research (Berlin, Germany) and the German Federal Ministry of Family Affairs, Senior Citizens, Women and Youth (Berlin, Germany). This work was supported by the Ministry of Culture and Science of the state of North Rhine-Westphalia (Düsseldorf, Germany) and the German Federal Ministry of Health (Berlin, Germany). This study was supported in part by a grant from the German Federal Ministry of Education and Research to the German Center for Diabetes Research (DZD). The funders of the study had no role in study design, data collection, analysis, interpretation or writing of the report.

Duality of interest The authors declare that there is no duality of interest associated with this manuscript.

Contribution statement $\mathrm{CN}, \mathrm{MCK}, \mathrm{CH}$ and $\mathrm{BS}$ designed the study. $\mathrm{HM}$ and MR contributed to the study design. $\mathrm{CN}, \mathrm{MCK}, \mathrm{BH}, \mathrm{HB}$ and $\mathrm{BS}$ contributed data. $\mathrm{CN}, \mathrm{MCK}, \mathrm{HM}$ and $\mathrm{CH}$ drafted the statistical analysis plan. HM performed the statistical analysis. CN, MCK and $\mathrm{CH}$ wrote the manuscript. All authors contributed to the discussion of the data, critically revised the manuscript for important intellectual content and approved the final version of the manuscript. $\mathrm{CH}$ is the guarantor of this work.

\section{References}

1. Yang RZ, Lee MJ, Hu H et al (2006) Identification of omentin as a novel depot-specific adipokine in human adipose tissue: possible role in modulating insulin action. Am J Physiol Endocrinol Metab 290:E1253-E1261

2. Herder C, Carstensen M, Ouwens DM (2013) Anti-inflammatory cytokines and risk of type 2 diabetes. Diabetes Obes Metab 15(Suppl 3):39-50

3. GTExPortal. Available from https://gtexportal.org/home/. Accessed $12 \mathrm{Feb} 2019$

4. de Souza Batista CM, Yang RZ, Lee MJ et al (2007) Omentin plasma levels and gene expression are decreased in obesity. Diabetes 56:1655-1661

5. Menzel J, di Giuseppe R, Biemann R et al (2016) Omentin-1 and risk of myocardial infarction and stroke: results from the EPICPotsdam cohort study. Atherosclerosis 251:415-421

6. Jung CH, Jung SH, Kim BY, Kim CH, Kang SK, Mok JO (2015) Association of serum omentin levels with cardiac autonomic neuropathy in patients with type 2 diabetes mellitus: a hospital-based study. Cardiovasc Diabetol 14:140

7. Vu A, Sidhom MS, Bredbeck BC, Kosmiski LA, Aquilante CL (2014) Evaluation of the relationship between circulating omentin-1 concentrations and components of the metabolic syndrome in adults without type 2 diabetes or cardiovascular disease. Diabetol Metab Syndr 6:4

8. Orlik B, Madej P, Owczarek A et al (2014) Plasma omentin and adiponectin levels as markers of adipose tissue dysfunction in normal weight and obese women with polycystic ovary syndrome. Clin Endocrinol (Oxf) 81:529-535

9. Saely CH, Leiherer A, Muendlein A et al (2016) High plasma omentin predicts cardiovascular events independently from the presence and extent of angiographically determined atherosclerosis. Atherosclerosis 244:38-43

10. Shibata R, Ouchi N, Kikuchi R et al (2011) Circulating omentin is associated with coronary artery disease in men. Atherosclerosis 219:811-814

11. Herder C, Ouwens DM, Carstensen M et al (2015) Adiponectin may mediate the association between omentin, circulating lipids and insulin sensitivity: results from the KORA F4 study. Eur J Endocrinol 172:423-432

12. Pan HY, Guo L, Li Q (2010) Changes of serum omentin-1 levels in normal subjects and in patients with impaired glucose regulation and with newly diagnosed and untreated type 2 diabetes. Diabetes Res Clin Pract 88:29-33

13. Yan P, Liu D, Long M, Ren Y, Pang J, Li R (2011) Changes of serum omentin levels and relationship between omentin and adiponectin concentrations in type 2 diabetes mellitus. Exp Clin Endocrinol Diabetes 119:257-263

14. Zhang Q, Zhu L, Zheng M et al (2014) Changes of serum omentin1 levels in normal subjects, type 2 diabetes and type 2 diabetes with overweight and obesity in Chinese adults. Ann Endocrinol (Paris) 75:171-175

15. Kataoka Y, Shibata R, Ohashi K et al (2014) Omentin prevents myocardial ischemic injury through AMP-activated protein kinase- and Akt-dependent mechanisms. J Am Coll Cardiol 63: $2722-2733$

16. Hiramatsu-Ito M, Shibata R, Ohashi K (2016) Omentin attenuates atherosclerotic lesion formation in apolipoprotein E-deficient mice. Cardiovasc Res 110:107-117

17. Watanabe K, Watanabe R, Konii H et al (2016) Counteractive effects of omentin-1 against atherogenesis. Cardiovasc Res 110:118-128 
18. Wittenbecher C, Menzel J, Carstensen-Kirberg M et al (2016) Omentin-1, adiponectin, and the risk of developing type 2 diabetes. Diabetes Care 39:e79-e80

19. Herder C, Kannenberg JM, Niersmann C et al (2017) Independent and opposite associations of serum levels of omentin- 1 and adiponectin with increases of glycaemia and incident type 2 diabetes in an older population: KORA F4/FF4 study. Eur J Endocrinol 177:277-286

20. Schöttker B, Herder C, Rothenbacher D et al (2013) Proinflammatory cytokines, adiponectin, and increased risk of primary cardiovascular events in diabetic patients with or without renal dysfunction: results from the ESTHER study. Diabetes Care 36:1703-1711

21. Looker HC, Colombo M, Agakov F et al (2015) Protein biomarkers for the prediction of cardiovascular disease in type 2 diabetes. Diabetologia 58:1363-1371

22. Schöttker B, Haug U, Schomburg L et al (2013) Strong associations of 25-hydroxyvitamin D levels with all-cause, cardiovascular, cancer and respiratory disease mortality in a large cohort study. Am J Clin Nutr 97:782-793

23. Raum E, Rothenbacher D, Low M, Stegmaier C, Ziegler H, Brenner H (2007) Changes of cardiovascular risk factors and their implications in subsequent birth cohorts of older adults in Germany: a life course approach. Eur J Cardiovasc Prev Rehabil 14:809-814

24. Schöttker B, Herder C, Muller H, Brenner H, Rothenbacher D (2012) Clinical utility of creatinine- and cystatin C-based definition of renal function for risk prediction of primary cardiovascular events in patients with diabetes. Diabetes Care 35:879-886

25. Herder C, Schöttker B, Rothenbacher D et al (2011) Interleukin-6 in the prediction of primary cardiovascular events in diabetes patients: results from the ESTHER study. Atherosclerosis 216:244-247

26. Inker LA, Eckfeldt J, Levey AS et al (2011) Expressing the CKDEPI (Chronic Kidney Disease Epidemiology Collaboration) cystatin $\mathrm{C}$ equations for estimating GFR with standardized serum cystatin C values. Am J Kidney Dis 58:682-684

27. Herder C, Bongaerts BW, Ouwens DM et al (2015) Low serum omentin levels in the elderly population with Type 2 diabetes and polyneuropathy. Diabet Med 32:1479-1483

28. Carstensen-Kirberg M, Kannenberg JM, Huth C et al (2017) Inverse associations between serum levels of secreted frizzledrelated protein-5 (SFRP5) and multiple cardiometabolic risk factors: KORA F4 study. Cardiovasc Diabetol 16:109

29. Herder C, Kannenberg JM, Carstensen-Kirberg M et al (2017) Serum levels of interleukin-22, cardiometabolic risk factors and incident type 2 diabetes: KORA F4/FF4 study. Cardiovasc Diabetol 16:17

30. Hansen CS, Vistisen D, Jørgensen ME et al (2017) Adiponectin, biomarkers of inflammation and changes in cardiac autonomic function: Whitehall II study. Cardiovasc Diabetol 16:153

31. Gu N, Dong Y, Tian Y et al (2017) Anti-apoptotic and angiogenic effects of intelectin-1 in rat cerebral ischemia. Brain Res Bull 130: $27-35$

32. Ohashi K, Shibata R, Murohara T, Ouchi N (2014) Role of antiinflammatory adipokines in obesity-related diseases. Trends Endocrinol Metab 25:348-355
33. Ouchi N, Ohashi K, Shibata R, Murohara T (2016) Protective roles of adipocytokines and myokines in cardiovascular disease. Circ $\mathrm{J}$ 80:2073-2080

34. Niersmann C, Hauck SM, Kannenberg JM et al (2019) Omentinregulated proteins combine a pro-inflammatory phenotype with an anti-inflammatory counterregulation in human adipocytes: a proteomics analysis. Diabetes Metab Res Rev 35:e3074

35. Zhong X, Zhang HY, Tan $\mathrm{H}$ et al (2011) Association of serum omentin-1 levels with coronary artery disease. Acta Pharmacol Sin 32:873-878

36. Shang FJ, Wang JP, Liu XT et al (2011) Serum omentin-1 levels are inversely associated with the presence and severity of coronary artery disease in patients with metabolic syndrome. Biomarkers $16: 657-662$

37. Wang XH, Dou LZ, Gu C, Wang XQ (2014) Plasma levels of omentin-1 and visfatin in senile patients with coronary heart disease and heart failure. Asian Pac J Trop Med 7:55-62

38. Harada K, Shibata R, Ouchi $N$ et al (2016) Increased expression of the adipocytokine omentin in the epicardial adipose tissue of coronary artery disease patients. Atherosclerosis 251:299-304

39. Yue J, Chen J, Wu Q et al (2018) Serum levels of omentin-1 association with early diagnosis, lesion volume and severity of acute ischemic stroke. Cytokine 111:518-522

40. Xu T, Zuo P, Wang Y, Gao Z, Ke K (2018) Serum omentin-1 is a novel biomarker for predicting the functional outcome of acute ischemic stroke patients. Clin Chem Lab Med 56:350-355

41. Moreno-Navarrete JM, Ortega F, Castro A et al (2011) Circulating omentin as a novel biomarker of endothelial dysfunction. Obesity (Silver Spring) 19:1552-1559

42. Kizer JR (2014) Adiponectin, cardiovascular disease, and mortality: parsing the dual prognostic implications of a complex adipokine. Metabolism 63:1079-1083

43. Wollert KC, Kempf T, Wallentin L (2017) Growth differentiation factor 15 as a biomarker in cardiovascular disease. Clin Chem 63: $140-151$

44. Herder C, de Las Heras Gala T, Carstensen-Kirberg M et al (2017) Circulating levels of interleukin 1-receptor antagonist and risk of cardiovascular disease: meta-analysis of six population-based cohorts. Arterioscler Thromb Vasc Biol 37:1222-1227

45. Herder C, Dalmas E, Böni-Schnetzler M, Donath MY (2015) The IL-1 pathway in type 2 diabetes and cardiovascular complications. Trends Endocrinol Metab 26:551-563

46. Herder C, Donath MY (2015) Interleukin-1 receptor antagonist: friend or foe to the heart? Lancet Diabetes Endocrinol 3:228-229

47. Fatkhullina AR, Peshkova IO, Dzutsev A et al (2018) An interleukin-23-interleukin-22 axis regulates intestinal microbial homeostasis to protect from diet-induced atherosclerosis. Immunity 49:943-957.e9

48. Herder C, Kowall B, Tabak AG, Rathmann W (2014) The potential of novel biomarkers to improve risk prediction of type 2 diabetes. Diabetologia 57:16-29

Publisher's note Springer Nature remains neutral with regard to jurisdictional claims in published maps and institutional affiliations. 